\title{
Machine Learning Techniques for Diagnosis of Lower Gastrointestinal Cancer: A Systematic Review
}

\author{
Mostafa Langarizadeh ${ }^{1}$, and Mohammadjavad Sayadi2,* \\ ${ }^{1}$ Health Management and Economics Research Center, Faculty of Health Management and Information Sciences, Iran University of Medical Sciences, Tehran, Iran \\ 2 Department of Health Information Management, Faculty of Health Management and Information Sciences, Iran University of Medical Sciences, Tehran, Iran
}

* Corresponding author: Mohammadjavad Sayadi, Department of Health Information Management, Faculty of Health Management and Information Sciences, Iran University of Medical Sciences, Tehran, Iran

Received 2020 December 08; Revised 2021 March 21; Accepted 2021 May 16

\begin{abstract}
Background: Nowadays, it can be seen that changes have taken place in the process of diseases and their clinical parameters. Accordingly, in some cases, general medical science and the use of clinical statistics based on the experiences of the physicians are not enough for the provision of sufficient tools for an early and accurate diagnosis. Therefore, medical science increasingly seeks to use unconventional methods and machine learning techniques. The issue of diagnosis in the medical world and the error rate of physicians in this regard are among the main challenges of the condition of patients and diseases. For this reason, in recent years, artificial intelligence tools have been used to help physicians. However, one of the main problems is that the effectiveness of machine learning tools is not studied much. Due to the sensitivity and high prevalence of diseases, especially gastrointestinal cancer, there is a need for a systematic review to identify methods of machine learning and artificial intelligence and compare their impact on the diagnosis of lower gastrointestinal cancers.

Objectives: This systematic review aimed to identify the machine learning methods used for the diagnosis of lower gastrointestinal cancers. Moreover, it aimed to classify the presented methods and compare their effectiveness and evaluation indicators.

Methods: This systematic review was conducted using six databases. The systematic literature review follows the Preferred Reporting Items for Systematic Reviews and Meta-Analyses statement for systematic reviews. The search strategy consisted of four expressions, namely "machine learning algorithm", "lower gastrointestinal", "cancer", and "diagnosis and screening", in that order. It should be mentioned that studies based on treatment were excluded from this review. Similarly, studies that presented guidelines, protocols, and instructions were excluded since they only require the focus of clinicians and do not provide progression along an active chain of reasoning. Finally, studies were excluded if they had not undergone a peer-review process. The following aspects were extracted from each article: authors, year, country, machine learning model and algorithm, sample size, the type of data, and the results of the model. The selected studies were classified based on three criteria: 1) machine learning model, 2) cancer type, and 3) effect of machine learning on cancer diagnosis.

Results: In total, 44 studies were included in this systematic literature review. The earliest article was published in 2010 , and the most recent was from 2019. Among the studies reviewed in this systematic review, one study was performed on the rectum (rectal cancer), one was about the small bowel (small bowel cancer), and 42 studies were on the colon (colon cancer, colorectal cancer, and colonic polyps). In total, 19 out of the 44 (43\%) articles from the systematic literature review presented a deep learning model, and 25 (57\%) articles used classic machine learning. The models worked mostly on image and all of them were supervised learning models. All studies with deep learning models used Convolutional Neural Network and were published between 2016 and 2019. The studies with classic machine learning models used diverse methods, mostly Support Vector Machine, K-Nearest Neighbors, and Artificial Neural Network.

Conclusion: Machine learning methods are suitable tools in the field of cancer diagnosis, especially in cases related to the lower gastrointestinal tract. These methods can not only increase the accuracy of diagnosis and help the doctor to make the right decision, but also help in the early diagnosis of cancer and reduce treatment costs. The methods presented so far have focused more on image data and more than anything else have helped to increase the accuracy of physicians in making the correct diagnosis. Achievement of the right method for early diagnosis requires more accurate data sets and analyses.
\end{abstract}

Keywords: Diagnosis, Lower gastrointestinal cancer, Machine learning

\section{Background}

Colorectal cancer is the third most common cancer in males and the second most common cancer in females. Unfortunately, a large number of patients with the final segment of the gastrointestinal (GI) system cancers die each year due to late diagnosis and overgrowth of cancer. One of the main reasons for this is that the symptoms of cancer do not appear until its later stages.

Today, due to the changes that have taken place in the process of diseases and their clinical parameters, in some cases, general medical science and the use of clinical statistics based on physician experience alone cannot provide sufficient tools for accurate and accurate diagnosis (1). Therefore, medical science increasingly seeks to use unconventional methods and machine learning techniques $(2,3)$. Based on the nature and the type of the problem that needs to be solved, as well as the used clinical data, supervised and unsupervised approaches to machine learning can be employed to diagnose diseases (4). Machine learning is one of the branches of artificial intelligence that enables a machine to study existing data without explicit planning to learn to discover patterns and make decisions based on existing data $(5,6)$. In other words, this technology allows the machine, like physicians, to describe and diagnose diagnostic data to 
discover and present meaningful structural features and determine which features are related to the results of the defined cases. This tool is very useful for processing the bulk of unstructured biometric data, such as radiological images (7). The issue of diagnosis in the medical world and the error rate of physicians in this regard is one of the main challenges due to the conditions of patients and diseases, and in recent years, artificial intelligence tools have been used to help physicians in this regard.

\section{Objectives}

One of the main issues is that the effectiveness of these tools is not much studied. Meanwhile, due to the sensitivity and high prevalence of diseases, especially gastrointestinal tract cancer, the need is felt for a systematic review to identify methods of machine learning in artificial intelligence and compare their impact on the diagnosis of lower gastrointestinal cancers.

\section{Methods}

\subsection{Protocol}

This systematic review follows the PRISMA statement for systematic reviews. Moreover, the Critical Appraisal Skills Program checklist was used to help the readers make sense of this qualitative research.

\subsection{Paper sources}

Publications from Google Scholar, Scopus, ProQuest, PubMed, Web of Science, Cochrane, and SID as a Persian database, were searched in September 2019 to identify articles that described and discussed the role of machine learning algorithms in the diagnosis of lower gastrointestinal cancers.

\subsection{Search strategy}

Due to the different terminologies of the selected databases in indexing papers, in an attempt to include all relevant articles, we used thesauruses, a systematic record in databases of subject headings used to index articles. To organize the search systematically, we grouped the search terms around four expressions: "Machine Learning", "Lower Gastrointestinal Cancers", "Cancer", and "Diagnosis and Screening". Further elaboration of the four expressions used to find eligible articles can be seen in Table 1. The search strategy consisted of four expressions: expression one (Machine Learning), expression two (Lower Gastrointestinal), expression three (Cancer), and expression four (Diagnosis and Screening). The terms within each expression were a mix of Medical Subject Headings (MeSH) terms and synonyms. We applied AND operator Between each expression and OR operator between each $\mathrm{MeSH}$ term and its synonyms. There were only a few exclusion criteria, such as being written in languages other than English and Persian, publication before 2010, and being performed during treatment and follow-up of patients.

\subsection{Inclusion and exclusion criteria of papers}

The focus of this study was on machine learning models used for diagnosing and screening lower gastrointestinal cancers. The focus was on studies that presented machine learning algorithms, models which were relevant for lower gastrointestinal cancers diagnosis. It should be mentioned that the studies which focused on treatment and diagnosis of non-cancer problems were excluded from this research. The studies that were published before 2010 and whose full text was unavailable or was not in English or Persian were excluded as well. Finally, studies were excluded if they had not undergone a peer-review process.

\subsection{Study selection and data extraction}

The OneNote 2016 was used to handle the articles. To remove duplicates in the identified references, the functions 'Find Duplicates' and 'Remove Duplicates' were applied. Titles and abstracts of the selected papers were read to find the eligible articles based on the inclusion and exclusion criteria. The full texts of the remaining articles were studied to extract the required data. The extracted data included authors, year, country, machine learning model and algorithm, sample size, type of data, and results of the model. To reduce bias during the selection and reviewing process, the author and one of the co-authors, went through each article systematically, discussed the scope of each article, and decided whether an article was relevant in proportion to the present systematic literature review. The inter-rater reliability was not calculated

\begin{tabular}{|c|c|c|c|}
\hline $\begin{array}{l}\text { Expression } 1 \\
\text { (Machine Learning) }\end{array}$ & $\begin{array}{c}\text { Expression 2 } \\
\text { (Lower Gastrointestinal) }\end{array}$ & $\begin{array}{l}\text { Expression } 3 \\
\text { (Cancer) }\end{array}$ & $\begin{array}{c}\text { Expression } 4 \\
\text { (Diagnosis and Screening) }\end{array}$ \\
\hline $\begin{array}{l}\text { Machine Learning OR } \\
\text { Artificial Intelligence OR } \\
\text { Deep Learning OR Neural } \\
\text { Networks OR Data Mining }\end{array}$ & $\begin{array}{l}\text { ileum OR large intestine OR jejunum OR colon } \\
\text { OR rectum OR cecum OR anal canal OR } \\
\text { Intestine Small OR Duodenum }\end{array}$ & $\begin{array}{l}\text { Neoplasm OR cancer } \\
\text { OR tumor }\end{array}$ & $\begin{array}{c}\text { Prediction OR diagnosis OR } \\
\text { detection OR screening OR } \\
\text { predict }\end{array}$ \\
\hline
\end{tabular}


in this study. The included machine learning models from the studies were subsequently described and classified according to the selected variables. The studies were classified in three ways: 1) type of machine learning model, 2) type of cancer, and 3) effect of machine learning on cancer diagnosis.

\section{Results}

\subsection{Study Selection}

Figure 1 shows the flowchart of the selection process of articles included in the systematic literature review. Systematic searches led to the identification of 774 articles. Before starting the preliminary screening process of titles and abstracts, 158 duplicates were removed; hence, 616 records remained to be screened. The screening process followed the inclusion and exclusion criteria as explained in the method section, leaving 74 articles for full-text review. There were 30 articles excluded based on the full-text review process; hence, the final number of studies included in the systematic review was 44 . The earliest relevant article was published in 2010, and the most recent was from 2019.

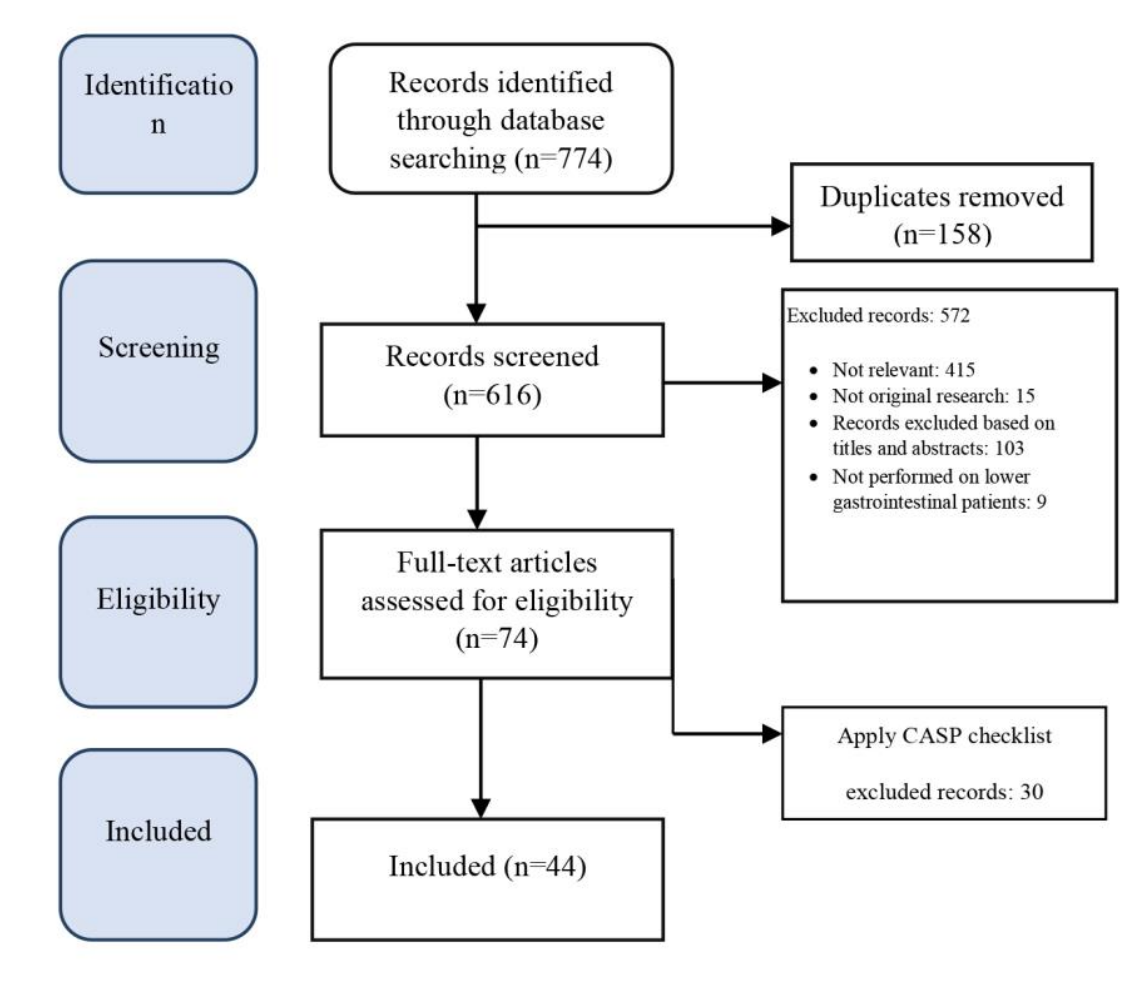

Figure 1. Flowchart of the selection process of the articles included in the systematic literature review. CASP: Critical Appraisal Skills Program

\begin{tabular}{|c|c|c|c|c|}
\hline Reference No. & Data presented in the article & Applied techniques & Year of publication & Country of publication \\
\hline (8) & Image & CNN & 2016 & UK \\
\hline (26) & Video & CNN & 2016 & Hong Kong \\
\hline (27) & Image & CNN & 2016 & Hong Kong \\
\hline (45) & Image & CNN & 2016 & USA \\
\hline (23) & Image & CNN & 2017 & Austria \\
\hline (28) & Image & CNN & 2017 & Taiwan \\
\hline (40) & Image & CNN & 2017 & UK \\
\hline (46) & Image & $\mathrm{CNN}+\mathrm{SVM}$ & 2017 & China \\
\hline (51) & Image & CNN & 2017 & Japan \\
\hline (30) & Image & CNN & 2018 & Germany \\
\hline (33) & Image & CNN & 2018 & USA \\
\hline (34) & Image & CNN & 2018 & China \\
\hline (29) & Image & CNN & 2019 & Japan \\
\hline (32) & Image & R-CNN & 2019 & USA \\
\hline (36) & Image \& Video & CNN & 2019 & Denmark \\
\hline (38) & Image & CNN & 2019 & Korea \\
\hline (39) & Image & CNN & 2019 & India \\
\hline (43) & Image & CNN & 2019 & France \\
\hline (44) & Image & CNN & 2019 & UK \\
\hline
\end{tabular}

CNN: convolutional neural network, SVM: support vector machine, R-CNN: region-based convolutional neural networks 


\begin{tabular}{|c|c|c|c|c|}
\hline Reference No. & Data presented in the article & Applied techniques & Year of publication & Country of publication \\
\hline (11) & Image & SVM & 2010 & USA \\
\hline (47) & Image & Fourier Filters & 2010 & Austria \\
\hline (12) & Image & SVM & 2011 & USA \\
\hline (13) & Image & GrayRLM & 2011 & Turkey \\
\hline (14) & Image & SVM & 2011 & Netherlands \\
\hline (48) & Image & $\mathrm{SVM}+\mathrm{KNN}+\mathrm{ANN}$ & 2011 & China \\
\hline (15) & Image \& Table & ANN & 2012 & Iran \\
\hline (16) & Image & RMM & 2012 & Turkey \\
\hline (18) & Image & ANN & 2012 & Italy \\
\hline (10) & Image & $\mathrm{SVM}+\mathrm{KNN}$ & 2013 & Korea \\
\hline (17) & Image & ANN & 2013 & USA \\
\hline (9) & Image & SVM & 2014 & Pakistan \\
\hline (19) & Image & SVM & 2014 & Taiwan \\
\hline (20) & Image & SVM & 2015 & Korea \\
\hline (21) & Image & Ensemble classifiers & 2016 & Spain \\
\hline (49) & Image & $\begin{array}{c}\text { J48, nearest neighbor, } \\
\text { backpropagation based on } \\
\text { multilayer perceptron, Naive } \\
\text { Bayes, and SVM }\end{array}$ & 2016 & Brazil \\
\hline (50) & Image & ANN & 2016 & Romania \\
\hline (22) & Image & $\begin{array}{l}\text { Binary pattern approach with } \\
\text { genetic fuzzy based improved } \\
\text { kernel SVM classifier }\end{array}$ & 2017 & USA \\
\hline (24) & Image & $\begin{array}{l}\text { Regression neural network } \\
\text { enhanced with the augmented } \\
\text { Lagrangian genetic algorithm }\end{array}$ & 2017 & India \\
\hline (25) & Image & SVM, MLP & 2017 & Turkey \\
\hline (35) & Image & $\begin{array}{c}\text { Sparse } \\
\text { autoencoder+SVM+image } \\
\text { processing methods }\end{array}$ & 2017 & China \\
\hline (31) & Image & SVM+dictionary learning & 2018 & Norway \\
\hline (37) & Image & Random forest & 2019 & China \\
\hline (41) & Image & SVM, deep belief network & 2019 & Turkey \\
\hline (42) & Image & SVM & 2019 & India \\
\hline
\end{tabular}

SVM: support vector machine, KNN: k-nearest neighbors, ANN: artificial neural network, RMM: resampling-based Markovian model, MLP: multilayer perceptron

\subsection{Types of Machine learning models \\ 4.2.1. Deep learning models}

In total, 19 out of the 44 (43\%) articles included in the systematic literature review presented a deep learning model. The oldest and newest of these studies were published in 2016 and 2019, respectively. The applied techniques in these 20 studies covered cancer diagnosis using the convolutional neural network (CNN). The data sets applied in these models encompassed various sizes and all of their data sets involved image and video
(Table 2).

\subsubsection{Classic machine learning models}

In total, $25(57 \%)$ of the reviewed studies used classic machine learning models. The oldest and newest studies were performed in 2010 and 2019, respectively. As it is shown in Table 3, these models mostly used support vector machine (SVM), artificial neural network (ANN), k-nearest neighbors (K-NN) as their techniques. In some studies, an ensemble model was used.

\begin{tabular}{|c|c|c|c|c|c|c|c|}
\hline Reference No. & Year & Country & Applied techniques & Accuracy (\%) & Sensitivity (\%) & Specificity (\%) & Other (\%) \\
\hline (47) & 2010 & Austria & Fourier filters & 96.9 & No & No & No \\
\hline (13) & 2011 & Turkey & GrayRLM & 87.1 & 79.2 & 90.7 & No \\
\hline (15) & 2012 & Iran & ANN & No & 85.7 & 44.4 & No \\
\hline (16) & 2012 & Turkey & $\begin{array}{c}\text { Resampling-based } \\
\text { Markovian model }\end{array}$ & 90.32 & No & No & No \\
\hline (10) & 2013 & Korea & $\begin{array}{l}\text { SVM } \\
\text { K-NN }\end{array}$ & 96.2 & No & No & No \\
\hline (9) & 2014 & Pakistan & SVM & 98.85 & 100 & 98 & No \\
\hline (21) & 2016 & Spain & $\begin{array}{l}\text { Ensemble } \\
\text { classifiers }\end{array}$ & 82.6 & 85.88 & 72.74 & No \\
\hline (46) & 2017 & China & $\mathrm{CNN}+\mathrm{SVM}$ & 98 & No & No & No \\
\hline (39) & 2019 & India & CNN & 72 & No & No & No \\
\hline (37) & 2019 & China & Random Forest & 91.8 & No & No & No \\
\hline (38) & 2019 & Korea & $\mathrm{CNN}$ & 94 & 100 & 88 & No \\
\hline (41) & 2019 & Turkey & $\begin{array}{c}\text { SVM, deep belief } \\
\text { network }\end{array}$ & 99.44 & 88.37 & No & F Score $=93.6$ \\
\hline
\end{tabular}

ANN: artificial neural network, SVM: support vector machine, K-NN: k-nearest neighbors, CNN: convolutional neural network 


\begin{tabular}{|c|c|c|c|c|c|c|c|}
\hline Reference No. & Year & Country & Applied techniques & $\begin{array}{c}\text { Accuracy } \\
(\%)\end{array}$ & $\begin{array}{c}\text { Sensitivity } \\
\text { (\%) }\end{array}$ & $\begin{array}{c}\text { Specificity } \\
\text { (\%) }\end{array}$ & Other (\%) \\
\hline (12) & 2011 & USA & SVM & No & 79 & 83 & No \\
\hline (17) & 2013 & USA & ANN & No & No & No & RSME $=1.26$ \\
\hline (8) & 2016 & UK & CNN & 78 & 82 & No & F1 Score $=80$ \\
\hline (27) & 2016 & Hong Kong & CNN & 85.9 & 87.6 & No & $\begin{array}{c}F 1 \\
\text { Score }=87.01\end{array}$ \\
\hline (49) & 2016 & Brazil & $\begin{array}{c}\text { J48, Nearest neighbor, } \\
\text { Backpropagation based on } \\
\text { multilayer perceptron, naive } \\
\text { Bayes and SVM }\end{array}$ & No & 92.54 & 85.07 & No \\
\hline (50) & 2016 & Romania & ANN & 84.52 & No & No & No \\
\hline (23) & 2017 & Austria & CNN & 96.4 & No & No & No \\
\hline (40) & 2017 & UK & CNN & No & No & No & F1 Score $=89$ \\
\hline (29) & 2019 & Japan & CNN & 81.2 & 89.0 & 67.5 & No \\
\hline (32) & 2019 & USA & CNN (R-CNN) & No & No & No & $\begin{array}{c}F 1 \\
\text { Score }=96.6\end{array}$ \\
\hline (43) & 2019 & France & CNN & No & No & No & $\begin{array}{c}\text { Dice } \\
\text { coefficient= } \\
0.93\end{array}$ \\
\hline (44) & 2019 & UK & CNN & No & No & No & $\begin{array}{c}\text { Dice } \\
\text { coefficient }=0 . \\
9\end{array}$ \\
\hline
\end{tabular}

RMSD: root-mean-square deviation, SVM: support vector machine, ANN: artificial neural network, CNN: convolutional neural network, RCNN: region-based convolutional neural networks

\begin{tabular}{|c|c|c|c|c|c|c|c|}
\hline Reference No. & Year & Country & Applied techniques & Accuracy (\%) & Sensitivity (\%) & Specificity (\%) & Other (\%) \\
\hline (11) & 2010 & USA & SVM & No & 83 & No & No \\
\hline (18) & 2012 & Italy & ANN & 100 & 100 & No & No \\
\hline (19) & 2014 & Taiwan & SVM & 96 & No & No & No \\
\hline (20) & 2015 & Korea & SVM & 70.67 & 70.6 & No & No \\
\hline (26) & 2016 & $\begin{array}{l}\text { Hong } \\
\text { Kong }\end{array}$ & CNN & 88.1 & 71 & No & $\begin{array}{c}\text { F1 } \\
\text { Score }=78.6\end{array}$ \\
\hline (45) & 2016 & USA & CNN & 99 & No & 98 & No \\
\hline (22) & 2017 & USA & SVM & 93.2 & No & No & No \\
\hline (24) & 2017 & India & $\begin{array}{l}\text { Regression neural } \\
\text { network }\end{array}$ & 97.37 & 96.5 & 96.25 & No \\
\hline (25) & 2017 & Turkey & $\begin{array}{l}\text { SVM } \\
\text { MLP }\end{array}$ & No & No & 95.8 & No \\
\hline (28) & 2017 & Taiwan & CNN & No & 87.1 & 96.3 & No \\
\hline (35) & 2017 & China & SVM & No & No & No & No \\
\hline (51) & 2017 & Japan & CNN & 72 & 81 & No & No \\
\hline (30) & 2018 & Germany & CNN & No & 93.9 & 89 & No \\
\hline (31) & 2018 & Norway & SVM & 95.9 & 95.8 & 95.9 & No \\
\hline (33) & 2018 & USA & CNN & 96.4 & No & No & No \\
\hline (34) & 2018 & China & CNN & No & 94.3 & 95.92 & No \\
\hline (36) & 2019 & Denmark & CNN & No & No & No & No \\
\hline (42) & 2019 & India & SVM & 95.7 & 95.4 & 96 & No \\
\hline
\end{tabular}

SVM: support vector machine, ANN: artificial neural network, CNN: convolutional neural network MLP: multilayer perceptron

\subsection{Types of cancer and the effect of machine learning} 4.3.1. Rectal Cancer

Among the studies reviewed in this systematic review, only one study was conducted on the rectom organ. In the aforementioned study, which predicted rectal cancer and used PET-CT images and the SVM method, in addition to the presence or absence of cancer, its physical characteristics were also identified. It should be mentioned that it was conducted in the Netherlands in 2011 (16). Notable points of this study are its only evaluation index which is the area under the curve, and it is not clear whether it has performed correctly in other possible indicators or not. In addition, there is no comparison with similar works in this study; therefore, its effectiveness cannot be commented on in this study.

\subsubsection{Small bowel}

Among the studies included in this systematic review, only one study was performed on the small bowel organ. In the above-mentioned study, which diagnosed a small bowel tumor and used endoscopic images and an ensemble method, an attempt was made to accurately identify the tumor in question. It was conducted in 2011 in China (48), and one of its notable points was its evaluation indicators. In the aforementioned study, accuracy, sensitivity, and specificity were used and acceptable results were obtained, compared to other models.

\subsubsection{Colon cancer}

Among the studies included in this systematic review, there were 42 studies on colon cancers. It is 
noteworthy that 12,11 , and 19 of these studies were related to the diagnosis of colon cancer, colorectal cancer, and cancerous polyps.

\subsubsection{Colon cancer}

i. According to the accuracy index, the highest value was related to the reference number (41) which was 99.44. In this study, published in 2019, the SVM method was used. Reviewing the studies, it can be seen that the average accuracy in the studies carried out during 2015-19 is higher than that of the studies performed during 2010-15. Moreover, the accuracy of the models implemented with the CNN method (on average) is higher than that of the other models.

ii. In terms of sensitivity index, two studies with reference numbers of (9) and (38) had the highest value which is $100 \%$. The first study was published in 2014 and used SVM, and the second one was published in 2019 and used CNN.

iii. The specificity index was reported in only five studies, the study with reference number (9) being the highest. (Table 4)

\subsubsection{Colorectal cancer}

i. According to the accuracy index, the highest value is related to reference number (23) which is 69.4. In this study, published in 2017, the CNN method was used. A review of the studies revealed that the accuracy of the models implemented with the CNN method (on average) is higher than that of the other methods.

ii. In terms of sensitivity index, the study with reference number (49) had the highest value which was $92.54 \%$. The aforementioned study was published in 2014 and used K-NN.

iii. The specificity index was reported in only three studies, the highest of which belonged to the study with reference number (12) which was published in 2011 and used SVM.

iv. Some studies have also reported the F1 Score among which the highest value (96\%) was related to the study with reference number (32) published in 2019. It should be noted that this study used CNN. (Table 5)

\subsubsection{Colonic Polyp}

i. According to the accuracy index, the highest value $(100 \%)$ is related to reference number (18). In this study, published in 2012, the ANN method was used. A review of the studies indicated that the accuracy of the models implemented with the SVM method (on average) is higher than that of the other models.

ii. In terms of sensitivity index, the highest value $(100 \%)$ was related to the study with reference number (18). This study was published in 2012 and used ANN. iii. The specificity index was reported in only three studies, and the study with reference number (45) had the highest index. The aforementioned research was published in 2016 and used CNN. (Table 6)

\section{Discussion}

Machine learning techniques are appropriate tools for the diagnosis of cancer, especially in cases involving the lower gastrointestinal tract. They not only increase the accuracy of the diagnosis and help the doctors make a better decision but also help in early cancer detection. The methods presented so far have focused more on the image as their dataset and increased the accuracy of the diagnosis of physicians. Achievement of the right method for early detection requires appropriate data sets and more accurate analysis.

By conducting this review, which has finally included 44 articles, the following has been achieved:

* Articles focused on the diagnosis of three types of cancer (Colon cancer, colorectal cancer, and polyp detection). The variety of models used in these studies was high and among them, SVM and CNN tools were the most commonly used methods. The SVM was usually used in the studies with a low volume of data, while CNN was used in situations where the volume of used data was high. Although this choice is closely related to the taste and ability of the researcher, in the case of data constraints, they were forced to choose despite this.

* Among the reviewed articles, only one article (15) dealt with the issue of early detection. In addition to being a potential for future research work, this can also be a threat. This means that no attempt has been made to create a data set for this issue. It is noteworthy that in the aforementioned study, in addition to image data, tabular data, including demographic data and patient records were used which are necessary for early diagnosis.

* It should be noted that the evaluations have not been performed based on a single procedure and each study has used its own indicators. For example, some studies have reported only one indicator; hence, it is not possible to know whether this study performed better in terms of other indicators or not. This can be a weakness but regarding the values reported for the indicators in these studies, accuracy and sensitivity indices were on average better in newer studies and studies that used the CNN method. Moreover, the specificity index was better reported in studies that have used the SVM method.

* Most studies concluded that the use of machine learning tools and especially methods based on CNN has contributed significantly to the accuracy and speed of diagnosis of physicians, especially in situations where the physician faces a large amount of video and video data. 


\section{Conclusion}

Machine learning methods are suitable tools in the field of cancer diagnosis, especially in cases related to the lower gastrointestinal tract. They can increase the accuracy of diagnosis, help the doctor to make the right decision, and also help in early diagnosis of cancer and reduction of treatment costs. The methods presented so far have focused more on image data and helped to increase the accuracy of the diagnosis of the physician more than anything else. Achievement of the right method for early diagnosis requires more accurate data sets and analyses.

\section{References}

1. Ghahramani Z. Probabilistic machine learning and artificial intelligence. Nature. 2015;521(7553):452-9. doi: 10.1038/ nature14541. [PubMed: 26017444].

2. Azimi P, Mohammadi HR, Benzel EC, Shahzadi S, Azhari S, Montazeri A. Artificial neural networks in neurosurgery. J Neurol Neurosurg Psychiatry. 2015;86(3):251-6. doi: 10.1136/jnnp-2014-307807. [PubMed: 24987050].

3. Deo RC. Machine learning in medicine Circulation. 2015;132(20):1920-30. doi: 10.1161/CIRCULATIONAHA. 115.001593. [PubMed: 26572668].

4. Senders JT, Staples PC, Karhade AV, Zaki MM, Gormley WB, Broekman MLD, et al. Machine learning and neurosurgical outcome prediction: a systematic review. World Neurosurg. 2018;109:476-86.e1. doi: 10.1016/j.wneu.2017.09.149. [PubMed: 28986230].

5. Johnson AE, Ghassemi MM, Nemati S, Niehaus KE, Clifton DA, Clifford GD. Machine learning and decision support in critical care. Proc IEEE Inst Electr Electron Eng. 2016;104(2):444-66. doi: 10.1109/JPROC.2015.2501978. [PubMed: 27765959].

6. Senders JT, Zaki MM, Karhade AV, Chang B, Gormley WB, Broekman ML, et al. An introduction and overview of machine learning in neurosurgical care. Acta Neurochir (Wien). 2018;160(1):29-38. doi: 10.1007/s00701-017-3385-8. [PubMed: 29134342].

7. Shameer K, Johnson KW, Glicksberg BS, Dudley JT, Sengupta PP. Machine learning in cardiovascular medicine: are we there yet? Heart. 2018;104(14):1156-64. doi: 10.1136/heartjnl2017-311198. [PubMed: 29352006].

8. Sirinukunwattana K, Ahmed Raza SE, Yee-Wah Tsang, Snead DR, Cree IA, Rajpoot NM. Locality sensitive deep learning for detection and classification of nuclei in routine colon cancer histology images. IEEE Trans Med Imaging. 2016;35(5):1196206. doi: 10.1109/TMI.2016.2525803. [PubMed: 26863654]

9. Rathore S, Hussain M, Aksam Iftikhar M, Jalil A. Ensemble classification of colon biopsy images based on information rich hybrid features. Comput Biol Med. 2014;47:76-92. doi: 10.1016/j.compbiomed.2013.12.010. [PubMed: 24561346].

10. Lee S, Kim K, Lee H, Jun $\mathrm{CH}$, Chung H, Park JJ. Improving the classification accuracy for IR spectroscopic diagnosis of stomach and colon malignancy using non-linear spectral feature extraction methods. Analyst. 2013;138(14):4076-82. doi: 10.1039/c3an00256j. [PubMed: 23687649].

11. Wang S, Yao J, Petrick N, Summers RM. Combining statistical and geometric features for colonic polyp detection in CTC based on multiple kernel learning. Int J Comput Intell Appl. 2010;9(1):1-15. doi: 10.1142/S1469026810002744. [PubMed: 20953299].

12. Rodriguez-Diaz E, Castanon DA, Singh SK, Bigio IJ. Spectral classifier design with ensemble classifiers and misclassification-rejection: application to elastic-scattering spectroscopy for detection of colonic neoplasia. J Biomed Opt. 2011;16(6):067009. doi: 10.1117/1.3592488. [PubMed: 21721830].

13. Tosun $\mathrm{AB}$, Gunduz-Demir C. Graph run-length matrices for histopathological image segmentation. IEEE Trans Med Imaging. 2011;30(3):721-32. doi: 10.1109/TMI.2010.2094200. [PubMed: 21097378]

14. van Stiphout RG, Lammering G, Buijsen J, Janssen $M H$, Gambacorta MA, Slagmolen P, et al. Development and external validation of a predictive model for pathological complete response of rectal cancer patients including sequential PET-CT imaging. Radiother Oncol. 2011;98(1):126-33. doi: 10.1016/j.radonc.2010.12.002. [PubMed: 21176986].

15. Biglarian A, Bakhshi E, Gohari MR, Khodabakhshi R. Artificial neural network for prediction of distant metastasis in colorectal cancer. Asian Pac J Cancer Prev. 2012;13(3):927-30. doi: 10.7314/apjcp.2012.13.3.927. [PubMed: 22631673].

16. Ozdemir E, Sokmensuer C, Gunduz-Demir C. A resamplingbased Markovian model for automated colon cancer diagnosis. IEEE Trans Biomed Eng. 2012;59(1):281-9. doi: 10.1109/TBME.2011.2173934. [PubMed: 22049357].

17. Bell CS, Obstein KL, Valdastri P. Image partitioning and illumination in image-based pose detection for teleoperated flexible endoscopes. Artif Intell Med. 2013;59(3):185-96. doi: 10.1016/j.artmed.2013.09.002. [PubMed: 24188575].

18. Bevilacqua V. Three-dimensional virtual colonoscopy for automatic polyps detection by artificial neural network approach: New tests on an enlarged cohort of polyps. Neurocomputing. 2013;116:62-75. doi: 10.1016/j.neucom.2012.03.026.

19. Fu JJ, Yu YW, Lin HM, Chai JW, Chen CC. Feature extraction and pattern classification of colorectal polyps in colonoscopic imaging. Comput Med Imaging Graph. 2014;38(4):267-75. doi: 10.1016/j.compmedimag.2013.12.009. [PubMed: 24495469].

20. Bae SH, Yoon KJ. Polyp detection via imbalanced learning and discriminative feature learning. IEEE Trans Med Imaging. 2015;34(11):2379-93. doi: 10.1109/TMI.2015.2434398. [PubMed: 26011864].

21. Mesejo P, Pizarro D, Abergel A, Rouquette O, Beorchia S, Poincloux L, et al. Computer-aided classification of gastrointestinal lesions in regular colonoscopy. IEEE Trans Med Imaging. 2016;35(9):2051-63. doi: 10.1109/TMI.2016.2547947. [PubMed: 28005009].

22. Geetha K, Rajan C. Heuristic classifier for observe accuracy of cancer polyp using video capsule endoscopy. Asian Pac J Cancer Prev. 2017;18(6):1681-8. doi: 10.22034/APJCP.2017.18.6.1681. [PubMed: 28670889].

23. Kainz P, Pfeiffer M, Urschler M. Segmentation and classification of colon glands with deep convolutional neural networks and total variation regularization. Peer J. 2017;5:e3874. doi: 10.7717/peerj.3874. [PubMed: 29018612] .

24. Sivaganesan D. Wireless distributive personal communication for early detection of collateral cancer using optimized machine learning methodology. Wirel Pers Communicat. 2017;94(4):2291-302. doi: 10.1007/s11277-016-3411-9.

25. Tulum G, Bolat B, Osman O. A CAD of fully automated colonic polyp detection for contrasted and non-contrasted CT scans. Int J Comput Assist Radiol Surg. 2017;12(4):627-44. doi: 10.1007/s11548-017-1521-9. [PubMed: 28101760].

26. Yu L, Chen H, Dou Q, Qin J, Ann Heng P. integrating online and offline three-dimensional deep learning for automated polyp detection in colonoscopy videos. IEEE J Biomed Health Inform. 2017;21(1):65-75. doi: 10.1109/JBHI.2016.2637004. [PubMed: 28114049].

27. Zhang R, Zheng Y, Mak TW, Yu R, Wong SH, Lau JY, et al. Automatic detection and classification of colorectal polyps by transferring low-level CNN features from nonmedical domain. IEEE J Biomed Health Inform. 2017;21(1):41-7. doi: 10.1109/JBHI.2016.2635662. [PubMed: 28114040].

28. Chen PJ, Lin MC, Lai MJ, Lin JC, Lu HH, Tseng VS. Accurate classification of diminutive colorectal polyps using computeraided analysis. Gastroenterology. 2018;154(3):568-75. doi: 10.1053/j.gastro.2017.10.010. [PubMed: 29042219].

29. Ito $\mathrm{N}$, Kawahira $\mathrm{H}$, Nakashima $\mathrm{H}$, Uesato $\mathrm{M}$, Miyauchi $\mathrm{H}$, Matsubara H. Endoscopic diagnostic support system for cT1b colorectal cancer using deep learning. Oncology. 2019; 96(1):44-50. doi: 10.1159/000491636. [PubMed: 30130758].

30. Renner J, Phlipsen H, Haller B, Navarro-Avila F, Saint-HillFebles Y, Mateus D, et al. Optical classification of neoplastic 
colorectal polyps - a computer-assisted approach (the $\mathrm{COACH}$ study). Scand J Gastroenterol. 2018;53(9):1100-6. doi: 10.1080/00365521.2018.1501092. [PubMed: 30270677].

31. Shin Y, Balasingham I. Automatic polyp frame screening using patch based combined feature and dictionary learning. Comput Med Imaging Graph. 2018;69:33-42. doi: 10.1016/j.compmedimag.2018.08.001. [PubMed: 30172091].

32. Sornapudi S, Meng F, Yi S. Region-based automated localization of colonoscopy and wireless capsule endoscopy polyps. Appl Sci. 2019;9(12):2404. doi: 10.3390/app9122404.

33. Urban G, Tripathi P, Alkayali T, Mittal M, Jalali F, Karnes W, et al. Deep learning localizes and identifies polyps in real time with 96\% accuracy in screening colonoscopy. Gastroenterology. 2018;155(4):1069-78.e8. doi: 10.1053/j.gastro.2018.06.037. [PubMed: 29928897].

34. Wang P, Xiao X, Glissen Brown JR, Berzin TM, Tu M, Xiong F, et al. Development and validation of a deep-learning algorithm for the detection of polyps during colonoscopy. Nat Biomed Eng. 2018;2(10):741-8. doi: 10.1038/s41551-018-0301-3. [PubMed: 31015647].

35. Yuan Y, Li D, Meng MQ, Yixuan Y. Automatic polyp detection via a novel unified bottom-up and top-down saliency approach. IEEE J Biomed Health Inform. 2018;22(4):1250-60. doi: 10.1109/JBHI.2017.2734329. [PubMed: 28783650].

36. Blanes-Vidal V, Baatrup G, Nadimi ES. Addressing priority challenges in the detection and assessment of colorectal polyps from capsule endoscopy and colonoscopy in colorectal cancer screening using machine learning. Acta Oncol. 2019;58(Suppl 1):S29-36. doi: 10.1080/0284186X.2019.1584404. [PubMed: 30836800].

37. He B, Ji T, Zhang H, Zhu Y, Shu R, Zhao W, et al. MRI-based radiomics signature for tumor grading of rectal carcinoma using random forest model. J Cell Physiol. 2019;234(11):20501-9. doi: 10.1002/jcp.28650. [PubMed: 31074022].

38. Kim J, Oh JE, Lee J, Kim MJ, Hur BY, Sohn DK, et al. Rectal cancer: toward fully automatic discrimination of T2 and T3 rectal cancers using deep convolutional neural network. Int J Imag Syst Technol. 2019;29(3):247-59. doi: 10.1002/ima.22311.

39. Ubale AA, Shikalpure SG. Colon cancer biopsy image analysis using deep learning. Int J Recent Technol Eng. 2019;8(2):56947. doi: $10.35940 /$ ijrte.B3202.078219.

40. Manivannan S, Li W, Zhang J, Trucco E, McKenna SJ. Structure prediction for gland segmentation with hand-crafted and deep convolutional features. IEEE Trans Med Imaging. 2018; 37(1):210-21. doi: 10.1109/TMI.2017.2750210. [PubMed: 28910760].

41. Sari CT, Gunduz-Demir C. Unsupervised feature extraction via deep learning for histopathological classification of colon tissue images. IEEE Trans Med Imaging. 2019;38(5):1139-49. doi: 10.1109/TMI.2018.2879369. [PubMed: 30403624].

42. Shanmuga Sundaram P, Santhiyakumari N. An enhancement of computer aided approach for colon cancer detection in WCE images using ROI based color histogram and SVM2. J Med Syst. 2019;43(2):29. doi: 10.1007/s10916-018-1153-9. [PubMed: 30612188].

43. Binder T, Tantaoui EM, Pati P, Catena R, Set-Aghayan A, Gabrani M. Multi-Organ gland segmentation using deep learning. Front Med (Lausanne). 2019;6:173. doi: 10.3389/ fmed.2019.00173. [PubMed: 31428614].

44. Graham S, Chen H, Gamper J, Dou Q, Heng PA, Snead D, et al. MILD-Net: minimal information loss dilated network for gland instance segmentation in colon histology images. Med Image Anal. 2019;52:199-211. doi: 10.1016/j.media.2018.12.001. [PubMed: 30594772].

45. Roth HR, Lu L, Liu J, Yao J, Seff A, Cherry K, et al. Improving computer-aided detection using convolutional neural networks and random view aggregation. IEEE Trans Med Imaging. 2016;35(5):1170-81. doi: 10.1109/TMI.2015.2482920. [PubMed: 26441412].

46. Xu Y, Jia Z, Wang LB, Ai Y, Zhang F, et al. Large scale tissue histopathology image classification, segmentation, and visualization via deep convolutional activation features. BMC Bioinformatics. 2017;18(1):281. doi: 10.1186/s12859-0171685-x. [PubMed: 28549410].

47. Häfner M, Brunauer L, Payer H, Resch R, Gangl A, Uhl A, et al. Computer-aided classification of zoom-endoscopical images using Fourier filters. IEEE Trans Inf Technol Biomed. 2010;14(4):958-70. doi: 10.1109/TITB.2010.2044184. [PubMed: 20371417].

48. Li B, Meng MQ, Lau JY. Computer-aided small bowel tumor detection for capsule endoscopy. Artif Intell Med. 2011; 52(1):11-6. doi: 10.1016/j.artmed.2011.01.003. [PubMed: 21353503].

49. Oliva JT, Lee HD, Spolaôr N, Coy CS, Wu FC. Prototype system for feature extraction, classification and study of medical images. Expert Syst Appl. 2016;63:267-83. doi: 10.1016/j.eswa.2016.07.008.

50. Ștefănescu D, Streba C, Cârțână ET, Săftoiu A, Gruionu G, Gruionu LG. Computer aided diagnosis for confocal laser endomicroscopy in advanced colorectal adenocarcinoma. PLoS One. 2016; 11(5):e0154863. doi: 10.1371/journal.pone.0154863. [PubMed: 27144985].

51. Komeda $\mathrm{Y}$, Handa $\mathrm{H}$, Watanabe $\mathrm{T}$, Nomura T, Kitahashi $\mathrm{M}$, Sakurai $\mathrm{T}$, et al. Computer-aided diagnosis based on convolutional neural network system for colorectal polyp classification: preliminary experience. Oncology. 2017;93(Suppl 1):30-4. doi: 10.1159/000481227. [PubMed: 29258081]. 